\title{
La ludicisation des pratiques d'écriture sur Internet : une étude des fanfictions comme dispositifs jouables
}

\section{Fanny Barnabé}

\section{(2) OpenEdition \\ Journals}

Édition électronique

URL : http://journals.openedition.org/sdj/310

DOI : $10.4000 /$ sdj.310

ISSN : 2269-2657

Éditeur

Laboratoire EXPERICE - Centre de Recherche Interuniversitaire Expérience Ressources Culturelles Education

\section{Référence électronique}

Fanny Barnabé, "La ludicisation des pratiques d'écriture sur Internet : une étude des fanfictions comme dispositifs jouables », Sciences du jeu [En ligne], 2 | 2014, mis en ligne le 24 octobre 2014, consulté le 29 mars 2021. URL : http://journals.openedition.org/sdj/310 ; DOI : https://doi.org/ $10.4000 /$ sdj. 310

Ce document a été généré automatiquement le 29 mars 2021.

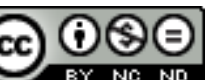

La revue Sciences du jeu est mise à disposition selon les termes de la Licence Creative Commons Attribution - Pas d'Utilisation Commerciale - Pas de Modification 4.0 International. 


\title{
La ludicisation des pratiques d'écriture sur Internet : une étude des fanfictions comme dispositifs jouables
}

\author{
Fanny Barnabé
}

\section{Gamification, ludicisation et numérique}

1 Si le concept de "gamification" connaît aujourd'hui une fortune certaine (dans le champ académique comme dans l'industrie) et s'il donne lieu à de nombreuses applications concrètes (qu'elles soient mercatiques, sociales ou éducatives), il n'éclaire que partiellement la propension actuelle du jeu à sortir de ses cadres habituels. Envisager la gamification comme " the use of game design elements in non-game contexts » (Deterding et al., 2011) permet bel et bien de montrer en quoi l'adjonction de certains éléments usuellement reconnus comme ludiques peut donner l'apparence d'un jeu à une situation qui n'est pas nécessairement jouable: ces «marqueurs pragmatiques de jouabilité » permettent, en d'autres termes, la transmission d'une «signification partagée» de jeu (Genvo, 2011). Toutefois, cette approche a le désavantage d'induire une conception «essentialisante » du ludique en le réduisant à une série de paramètres présentés comme objectivables (le score, le système de récompenses, la progression par niveaux, etc.). Elle occulte donc, d'une part, la diversité et la variabilité des représentations possibles de ce qu'est un jeu et, d'autre part, la primauté de «l'attitude ludique » de l'utilisateur (Henriot, 1989) sans laquelle le jeu ne peut advenir (et qui parfois suffit à « faire jeu»).

2 Or cette dernière caractéristique semble essentielle à la compréhension du ludique et de la manière dont il se diffuse. En effet, comme l'ont souligné Mathieu Triclot (2011), à travers la définition des « play studies », et Sébastien Genvo (2008), par l'idée de " play design », le jeu n'est pas tant la propriété d'un objet clos et fini qu'une expérience chaque 
fois singulière et susceptible de déborder du cadre sur lequel elle se construit. Cette conception a l'avantage de ne pas cloisonner la notion de jeu à l'intérieur des dispositifs qui lui sont traditionnellement associés et de permettre l'appréhension de pratiques ludiques "périphériques». Selon cette perspective, la «ludicisation» - définie par Genvo comme l'ensemble des « processus qui consistent à faire entrer un objet dans le monde du jeu» (2011, p. 62) - peut être envisagée comme une composante naturelle du jeu revenant à transposer d'un objet à l'autre une même attitude de joueur. La « jouabilité » des structures ludicisées dépendrait alors, selon Genvo (2011), de leur capacité de s'adapter à ce comportement et à le nourrir. En répondant à l'activité libre $\mathrm{du}$ joueur, les dispositifs deviennent donc de véritables games, c'est-à-dire des représentations temporairement figées d'un play en constante reconfiguration.

Cependant, si la ludicisation est un aspect propre au jeu en tant que play, l'importance croissante qui lui est prêtée trouve son explication dans le rôle de catalyseur qu'y joue aujourd'hui le numérique. Un certain nombre de chercheurs ${ }^{1}$ semblent en effet s'accorder sur l'existence d'une jouabilité intrinsèque à ce type de support ; autrement dit, d'un fort potentiel d'adaptation à l'attitude ludique de l'utilisateur. Cette « ludogénéité » du numérique (Vial, 2012) a pour conséquence de rendre perméables les frontières du " cercle magique $»^{2}$ : elle facilite la diffusion du play sur différents objets sémiotiques qui doivent alors s'adapter à l'appropriation ludique à laquelle ils sont soumis.

\section{Les pratiques d'écriture amateur sur Internet}

Dans le prolongement du cadre théorique qui vient d'être défini, ce travail vise à étudier le rôle d'activateur que joue le numérique dans les phénomènes de ludicisation (au sens d'attitude ludique généralisée et de réappropriation ludique d'objets qui ne sont initialement pas des jeux). Pour ce faire, nous nous baserons sur un cas de figure concret: la ludicisation des pratiques d'écriture amateur à travers le phénomène des fanfictions diffusées sur Internet.

5 Les fanfictions sont, comme leur nom l'indique, des récits fictionnels écrits par les fans et qui s'inspirent d'œuvres préexistantes (qu'elles soient littéraires comme la saga Harry Potter, cinématographiques comme Star Wars, ou encore vidéoludiques tel que nous le verrons plus loin). Ces récits peuvent poursuivre différents objectifs : prolonger un univers en comblant les failles laissées par l'auteur, détourner humoristiquement une histoire connue, mettre en scène des personnages appréciés au sein de réalités inédites, etc. Si la pratique existait avant l'avènement d'Internet (ce premier état de fait a été amplement décrit par Henry Jenkins, 1992 et 2006b), elle ne se développait alors que dans des communautés relativement confidentielles, et les œuvres produites ne bénéficiaient que d'une diffusion très restreinte (à travers l'échange de fanzines lors de conventions). La migration des fanfictions sur Internet, a considérablement accru la portée et la visibilité du phénomène en facilitant la production des textes et en permettant leur distribution à plus grande échelle. Cet accroissement n'en a toutefois pas été la seule conséquence: l'investissement du support numérique a également entraîné d'importantes modifications dans l'organisation de l'activité. Il est désormais possible, par exemple, de publier un texte progressivement (sans l'avoir préalablement terminé), de le modifier a posteriori, de laisser des commentaires (ou «reviews») à chaque étape d'une fiction et d'en influencer l'élaboration. Le coût d'entrée dans la 
communauté a d'autre part amplement diminué (n'importe qui peut publier un texte), si bien que l'apprentissage y a pris une place centrale (les compétences linguistiques ou scénaristiques ne sont plus tant un prérequis qu'un objectif). En outre, la multiplication des textes a modifié les modalités de leur consommation : pour faire le tri dans le vaste ensemble de fanfictions qui leur sont proposées, les lecteurs ont aujourd'hui la possibilité d'utiliser différents filtres au sein de leur recherche (sélection par genre, par personnage, etc.). ${ }^{3}$ Plus généralement, on peut supposer que la « ludogénéité » propre au numérique n'a pas été sans effet sur cette forme d'écriture.

6 Selon notre hypothèse, il semblerait que le passage du papier à Internet (induisant l'assimilation des codes du second support) a permis aux fanfictions de gagner en jouabilité et ce à plusieurs niveaux. Nous tâcherons de montrer qu'en soutenant l'«attitude ludique " des utilisateurs, le numérique a favorisé une réappropriation ludique de l'écriture amateur. L'on peut ainsi observer, dans les textes produits comme dans les structures qui les encadrent, un certain nombre de "marqueurs » signifiant l'usage ludique qui en est fait et qui fonctionnent comme autant d'invitations à entrer dans le «jeu» de l'écriture. Ce travail interrogera également la manière dont les fanfictions répondent au play qui les investit et alimentent l'expérience ludique. Leur jouabilité sera abordée à travers trois axes : un axe communautaire (les sociabilités qui entourent la pratique des fanfictions permettent-elles l'émergence du jeu ?), un axe portant sur les usages (les modalités d'écriture et de lecture sont-elles jouables ?) et un axe formel (les textes produits contiennent-ils des "marqueurs pragmatiques" (Genvo, 2011) du jeu ?).

7 Dans le but de refuser toute essentialisation du jeu, il importe toutefois de préciser, à ce stade, que la «jouabilité » propre aux fanfictions n'est pas envisagée ici comme un attribut définitif, mais plutôt comme une dynamique. Les textes écrits par les fans résultent de la réappropriation ludique d'un univers fictionnel (d'un play) qui se solidifie en nouveau système de règles (en game): l'écriture de fanfiction est effectivement soumise à certaines normes. Cependant, ce game est lui-même constamment renégocié par l'utilisation qu'en font les praticiens (les règles ne cessent d'être discutées, transgressées, réinventées). C'est donc tantôt par la normalisation de leurs enjeux et tantôt par leur ouverture à une appropriation libre que les fanfictions incitent au jeu - la tension qui oppose ces deux dimensions les empêchant d'être définitivement associées à tel ou tel aspect de la pratique (l'écriture ou la lecture, par exemple).

Outre l'intérêt que peuvent comporter ces questionnements pour la compréhension de la manière dont un objet initialement non ludique s'intègre dans une logique de jeu, le fait d'appréhender les fanfictions comme les supports d'une expérience ludique permet de résoudre une difficulté méthodologique propre à cet objet. En effet, étudier ces textes à l'aune de critères littéraires a pour conséquence, comme le note Francesca Coppa (2006), de présenter comme des défauts certaines caractéristiques fondamentales du genre (l'absence de linéarité, l'omniprésence des répétitions, les libertés prises avec la cohérence scénaristique, etc.). L’analyse sociologique traditionnelle, quant à elle, invite à ne voir ces pratiques "que sous le mode de la compensation culturelle» (François, 2010, p. 6), ce qui a pour effet de dissimuler les résistances que les communautés de fans opposent usuellement aux valeurs culturelles traditionnelles. Comme le souligne Philippe Le Guern, «[...] le rapport cultiste aux œuvres est une forme d'inversion de la valeur traditionnelle et de valorisation des 
éléments les moins "recommandables" " (Le Guern, 2002, p. 21). Cette compréhension nécessairement évaluative ne rend compte que très imparfaitement du phénomène en raison du conflit qu'elle génère entre deux communautés interprétatives. Les fanfictions se prêtent mal aux outils du théoricien littéraire, car ce dernier n'est pas leur « lecteur modèle »: elles s'adressent à un autre type de lecteur, capable, principalement, d'évaluer la portée de ces textes dans une architecture plus large (un univers fictionnel cohérent ou même, plus généralement, la culture populaire dans son ensemble). ${ }^{4}$ Envisager les fanfictions à travers le paradigme théorique du play permet, à l'inverse, de réduire cette distance et de formaliser, sans les trahir, les enjeux du domaine (telle fanfiction est-elle un bon support de l'attitude ludique? tel texte est-il «jouable»? etc.).

\section{Remarques méthodologiques}

Dans le but d'envisager les connexions entre fanfictions et jeu à travers leurs actualisations les plus riches, cette recherche s'est fondée sur l'analyse de textes s'inspirant de l'univers d'un jeu vidéo. Le corpus envisagé se compose de trente fanfictions basées sur la série de jeux la plus sujette à ce type de réappropriations, à savoir Kingdom Hearts. ${ }^{5}$ Les différents textes (dont la longueur totale s'élève à un peu plus de deux millions de mots) ont été prélevés sur le site Fanfiction.net ${ }^{6}$ en suivant deux critères : la langue (seules les fictions en français ont été considérées) et la popularité (les textes sélectionnés sont ceux qui ont été le plus souvent enregistrés comme " favoris " par les utilisateurs; en conséquence, ils sont censés être plus représentatifs de la pratique telle qu'elle doit être idéalement menée). L'analyse ne s'est pas bornée à la forme et au contenu des fanfictions, mais a également pris en compte les discours de leurs producteurs et consommateurs ainsi que les indices relevant du fonctionnement de la communauté qui transparaissaient dans les divers éléments du paratexte (la présentation du texte dans l'espace alloué par le site, les annotations de l'auteur insérées en début et en fin de chaque chapitre et les commentaires des lecteurs).

Cette nécessaire limitation du corpus peut avoir sur les résultats de l'étude un impact qu'il convient de garder à l'esprit. Toutefois, la comparaison de la présente analyse avec d'autres plus anciennes ${ }^{7}$ portant sur des « fandoms $»^{8}$ non vidéoludiques invite à penser que les conclusions ici présentées ne se limitent pas au seul cas de Kingdom Hearts mais sont généralisables à la pratique de la fanfiction dans son ensemble, indépendamment de l'œuvre qui lui sert de support. Par ailleurs, le présent article ne se penche pas tant sur le contenu des textes que sur les usages dont ils sont les sources, les résultats ou les témoins.

Dans le but d'alléger la présentation des sources, nous observerons dorénavant les conventions suivantes : sauf mention contraire, les sites référencés ont été consultés pour la dernière fois le 14 février 2012 et les fanfictions citées sont toutes issues de Fanfiction.net. 


\section{Une communauté jouable et la posture du fan-auteur : entre réflexivité et distanciation}

12 Avant d'interroger les conséquences de la ludicisation sur les modalités d'écriture de fanfictions et sur la forme des textes produits, il importe de montrer en quoi l'attitude des amateurs vis-à-vis de leur pratique peut être comparée à un play généralisé. Sur la base de l'analyse du fandom de Kingdom Hearts mais aussi d'enquêtes précédemment réalisées ${ }^{9}$, ce premier axe vise à mettre au jour les différents aspects de l'attitude des fans qui participent à inclure l'écriture de fanfiction dans une logique de jeu. Par rapport au champ littéraire traditionnel, cette pratique développe en effet une représentation singulière de la figure de l'auteur et de son rapport à l'œuvre.

13 Le principe même qui sous-tend la production de fanfictions entraîne une redéfinition de la figure du créateur et du droit de propriété intellectuelle qui lui est habituellement alloué. D'une part, la publication sur Internet permet à n'importe qui de devenir écrivain (ce qui participe à désacraliser le titre) et, d'autre part, le fait que les fans s'autorisent à réinvestir et à détourner des textes, des films, des bandes dessinées ou des jeux qui ne leur appartiennent pas présente ces œuvres comme des biens communs : « sur Internet, les frontières du texte se dissolvent, et avec elles l'autorité (devenue insupportable) de l'auteur » (Loveluck, 2008, p. 156). Le rôle du «bêta-lecteur " est révélateur de cette tendance : ce lecteur-critique a effectivement pour fonction de corriger les fanfictions avant leur publication. En influant directement sur la phase de création, il réfute donc l'autorité de l'auteur sur son œuvre (Karpovich, 2006, p. 176) ${ }^{10}$.

Dans le prolongement de ces mutations, les "auteurs secondaires» que sont les producteurs de fanfictions ont, quant à eux, tendance à adopter une posture très distanciée par rapport à leur activité, posture qui n'est pas sans rappeler les différentes définitions de l'attitude ludique. Le play est en effet tantôt décrit comme un "procès métaphorique " (Henriot, 1989, p. 300), tantôt comme un acte de "métacommunication » (le joueur doit superposer à son action le message « ceci est un jeu »; Bateson, 1977) ou comme une interaction au second degré avec le réel (le « faire comme si » de Schaeffer, 1999). La ludicisation de l'écriture des fanfictions passe donc, en première instance, par la mise à distance ludique de la pratique et des statuts auxquels elle devrait pouvoir faire accéder. Cette posture qui permet de «jouer à l'auteur », de demeurer au sein du «cercle magique» (Huizinga, 1950), comporte trois dimensions : le second degré, la réflexivité et la « fictionnalisation ».

\section{Une pratique « non sérieuse »}

15 Le statut du fan-écrivain se caractérise par une profonde ambivalence. En effet, si les auteurs de fanfictions s'investissent avec la plus grande application dans leur pratique, dans la vie de la communauté et même dans l'élaboration d'un métadiscours sur les textes produits ${ }^{11}$, ils ne cessent pas pour autant de se définir avant tout comme des fans. En d'autres termes : « conformément à l'éthos du fan "qui joue le jeu sérieusement sans se prendre au sérieux", ces auteurs amateur ne semblent pas nourrir d'ambitions démesurées " (Le Guern, 2002, p. 199). Ce positionnement a un impact direct sur la définition de la fanfiction: "[...] stories can only be defined as fan fiction if they originate in a self-identified fan culture, implying that the only fanfic is that body of work that explicitly labels 
itself "fanfic" - or at the least is composed by people who self-identify as fans " (Derecho, 2006, p. 62). Cet état de fait n'empêche pas nombre de ces écrivains amateurs d'envisager une réelle carrière d'auteur parallèlement à leur passe-temps. ${ }^{12}$ Néanmoins, l'activité menée en tant que fan semble très peu connectée à ces ambitions professionnelles : loin de chercher à inclure leur pratique dans un système plus légitime, les "fanficcers" refusent le plus souvent les tentatives de réappropriation qui sortent du cadre de la culture populaire dans laquelle ils s'inscrivent.

La gratuité (voire l'illégalité) de ces écrits en vient d'ailleurs, parfois, à être revendiquée comme une composante définitoire du domaine. En témoignent les réactions de méfiance exprimées vis-à-vis du récent Kindle Worlds (une plateforme de diffusion gérée par Amazon dont le but revendiqué est de permettre à certains auteurs de fanfictions d'être rémunérés sans nuire aux ayants-droits). En réponse au sujet «Fanfictions payantes sur Amazon» sur le forum Parlons fanfictions, un participant écrit :

Je ne vendrai ni n'achèterai, pour ma part. Je trouve ça totalement contraire au principe de la fanfiction. [...] Oui, certains ont fait de leur art un métier, que ce soit dans l'écriture, la musique, le cinéma, la peinture, etc. Mais la fanfiction est quand même une démarche différente du reste de l'écriture [...] («Fanfictions payantes sur Amazon ", forum Parlons fanfictions). ${ }^{13}$

17 La résistance aux détournements commerciaux s'explique, en partie, par la crainte de se voir imposer des règles et des limitations qui n'avaient pas cours au sein du « cercle magique » (la possibilité de mêler différents univers ou celle d'aborder des thèmes qui ne sont pas pour tous les publics est, pour ne citer que ces exemples, refusée aux auteurs qui diffusent leurs textes via Kindle Worlds). L'autonomie du champ fanfictionnel (notamment favorisée par l'investissement du support Internet) comporte, en somme, une forte dimension permissive :

[Fans] operate in a gift economy [...]. Being free of the commercial constraints that surround the source texts, they gain new freedom to explore themes or experiment with structures and styles that could not be part of the "mainstream" versions of these worlds (Jenkins, 2006a, pp. 180-181).

18 Toutefois, le refus des ingérences extérieures et l'exigence de gratuité (au sens large du terme) ne se limite pas au domaine marchand. En effet, contrairement aux autres figures d'écrivains amateurs qui, faute de pouvoir faire leur entrée dans le champ littéraire, développent parallèlement à celui-ci un "espace de consolation » qui en imite les structures (Poliak, 2006), les auteurs de fanfictions semblent plutôt s'affranchir des modèles fournis par la littérature traditionnelle. Une trop grande recherche formelle, une souscription aux genres légitimes ou l'élaboration au premier degré d'un éthos d'écrivain risquent, au contraire, d'être mal perçues par la communauté. Ainsi peut-on lire le commentaire d'une fan-auteure populaire qui, dans l'en-tête de son deuxième chapitre, s'excuse presque auprès de ses lecteurs pour avoir été trop " poétique »: «Second chapiiitre. $\mathrm{n} \_n$ Avec moins de descriptions poétiques (je vous épargne XD) et plus d'action » (Crushcrushcrush, chapitre 2).

19 De même, les représentations sacralisantes de l'activité littéraire dégagées par Claude Poliak auprès des écrivains amateurs - l'écriture répondrait à un besoin, une nécessité transcendantale, elle nécessiterait des lieux, des outils et des circonstances particulières, etc. - ne trouve guère d'écho dans les témoignages recueillis par Élodie Oger. Parmi ceux-ci, les motifs les plus fréquemment exprimés pour justifier le passage à l'écriture sont l'amour de l'œuvre originale (le plaisir d'y explorer de nouvelles 
pistes), le sentiment d'appartenir à une communauté, le désir d'apprentissage (progresser en écriture) et le divertissement (on écrit pour s'amuser).

En somme, si les fanfictions s'apparentent à la littérature, l'écriture n'y est pas moins «[transposée] sur un terrain à peu près libre de toute autorité culturelle, c'est-à-dire sur lequel les fans peuvent se constituer un capital et une compétence spécifiques » (Le Guern, 2002, p. 211). Le refus des réappropriations « sérieuses» de l'activité témoigne en partie de l'attitude ludique qui préside à son exécution : celle-ci se doit de rester sans conséquences sur le « monde réel».

\section{Distanciation par la réflexivité}

21 Le recul à l'égard de la pratique peut aussi passer par la réflexivité et par la mise à distance de l'objet même de l'engouement. Comme le souligne Sébastien François (2009, p.182), l'attitude des fans oscille constamment entre enthousiasme et critique: si l'écriture de fanfictions se fonde initialement sur une passion fervente, les auteurs s'évertuent parallèlement à se distinguer de la figure du " fanboy " (l'amateur exalté qui s'investit sans recul dans l'objet de son affection) et à éviter l'écueil du «Mary Sue " (l'introduction, dans une fiction, d'un personnage représentant une version idéalisée de l'auteur $\left.{ }^{14}\right)$. « [...] Cette double posture d'engagement (dans le collectif) et de distanciation (par rapport aux fans les plus "pris au jeu") leur permet en quelque sorte de jouer sans être totalement dupe du jeu, de se comporter comme fans sans être fanatiques " (Le Guern, 2002, p. 189). En découlent donc des stratégies complexes de représentation de soi qui, toujours selon Le Guern, trouvent leur expression privilégiée dans le jeu. Citons, pour exemple, la fanfiction humoristique et réflexive nommée $L a$ genèse $d u Y Y_{a i}{ }^{15}$, dans laquelle l'auteure se présente comme une "fangirl» tout en développant vis-à-vis de cette figure une posture d'ironie. En outre, le fait que ce discours réflexif sur le fonctionnement de la communauté prenne place au sein d'un texte publié (et non d'un forum, par exemple) met au jour l'existence d'une certaine perméabilité entre métadiscours et fiction (la réflexivité reste dans le domaine du « faire comme si » $)^{16}$, ce qui nous amène au point suivant.

\section{Distanciation par la fiction}

La «fictionnalisation de soi» constitue, en effet, une stratégie récurrente de distanciation utilisée par les auteurs. L'investissement du support numérique a eu pour conséquence d'anonymiser les rapports entre amateurs et de rendre possible la construction libre de sa propre représentation textuelle. Les acteurs de la communauté doivent dorénavant choisir un pseudonyme et élaborer un profil, à travers lesquels se dessine un véritable "personnage d'auteur" (dont le Mary sue n'est que l'aboutissement). Il existe d'ailleurs une parenté relativement perceptible entre l'écriture de fanfictions et le jeu de rôle (Cristofari, 2010, p. 2) : non seulement les jeux de rôle textuels prenant les fanfictions pour modèles foisonnent sur les forums (voir par exemple "Kingdom Hearts Forums»), mais certains auteurs admettent également s'inspirer de la manière dont ils jouent un personnage en roleplay pour le décrire par la suite dans une fiction : "J'ai casé mes envies de rp dans ce UA et...Roxas est égal à luimême comme je le joue en rp, enfin presque XD » (Seconde Chance, chapitre 2$)^{17}$ affirme l'un d'eux. 
Sans aller jusqu'à la construction d'un Mary sue, d'un avatar explicite, les auteurs investissent fréquemment leurs différents paratextes pour se mettre en scène comme des personnages existant aux marges de la fiction. Ainsi, il n'est pas rare de les voir, dans la présentation d'un chapitre, se représenter en train d'interagir avec un de leurs personnages ou simuler certaines actions par l'utilisation d'onomatopées, de smileys ou de signes typographiques comme l'atteste ce commentaire fourni à la suite du premier chapitre de Kokoro ga nai où l'auteure converse avec ses personnages sur ce qu'il vient de se passer dans l'histoire :

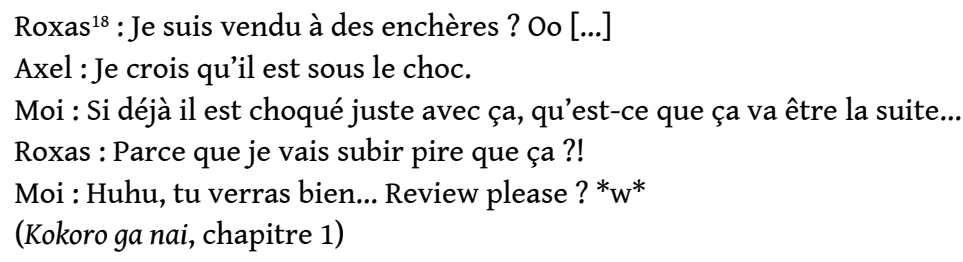

Ou encore, comme l'exemplifie cette présentation d'un chapitre où l'auteure annonce les résultats d'un sondage interrogeant les lecteurs sur leurs couples préférés :

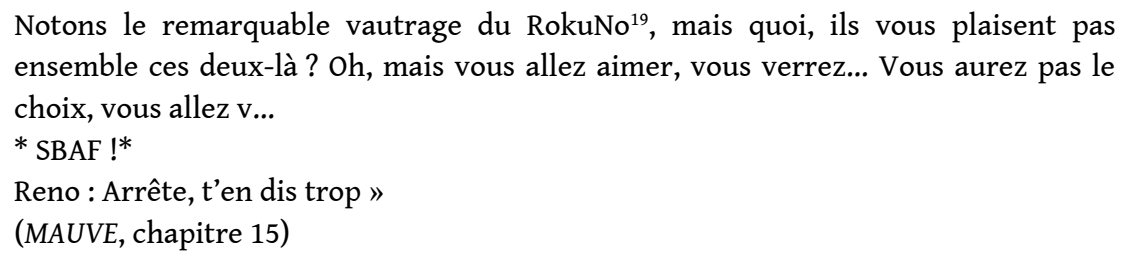

L'attitude des auteurs de fanfiction vis-à-vis de leur statut, de leurs productions ou de la pratique même de l'écriture se caractérise, en somme, par un important second degré qui apparaît comme un élément essentiel au bon fonctionnement de la communauté.

\section{Légitimation et sanction : un jeu aux rôles interchangeables}

Un aspect distinguant le champ fanfictionnel du champ littéraire et pouvant signifier l'usage ludique qui en est fait reste à évoquer: il s'agit du rapport d'égalité et d'interchangeabilité qui y relie auteurs et lecteurs. En effet, « contrairement au monde de l'art qui distingue l'artiste du critique, le monde de l'amateur entremêle les positions, si bien que production et discours, création et jugement ne sont jamais totalement séparés » (Flichy, $2010: 11)$. Les fans assument alternativement différentes fonctions au sein de la communauté (celles d'auteur, de lecteur, de critique ou même d'éditeur) et cette réversibilité des statuts entraîne un partage plus ou moins équitable de la légitimité : l'auteur n'est pas propriétaire de son œuvre et le lecteur n'a pas le monopole de la sanction.

L'absence de hiérarchie et l'échange des rôles rapprochent le domaine des fanfictions d'un espace de jeu, dans le sens où ils réduisent le coût d'entrée dans la communauté (réduction qui, on l'a vu, est également tributaire de l'utilisation d'Internet comme moyen de publication). Lorsqu'un auteur soumet un texte à la critique, il s'adresse avant tout à des pairs qui sont aussi investis affectivement que lui dans la pratique et dans le contenu des œuvres. Cet "entre-soi» et ce refus de la fixité des statuts permettent notamment aux praticiens de mettre la notion d'apprentissage au centre de l'activité : comme le souligne Patrice Flichy (2010, p. 90), la progression tend à être davantage valorisée que l'œuvre produite. L'importance de cette dimension se marque, 
par exemple, dans les nombreux outils et conseils qui sont fournis aux auteurs par les sites ou par leurs pairs ${ }^{20}$. Une certaine tolérance par rapport aux erreurs se dégage également des discours des amateurs, comme en témoignent les différentes réponses à cette auteure qui demandait pourquoi «poster des fan fictions quand on ne sait pas écrire?» :

Il y a [...] ceux qui sont conscients de faire des fautes, et qui se disent que le meilleur moyen pour apprendre à se corriger, c'est d'écrire [...]. Je parle toujours par expérience personnelle, mais j'ai par exemple eu une lectrice très gentille qui me signalait mes fautes récurrentes pour que j'y fasse attention par la suite.

La fanfiction est une activité de fan, pour moi, qui relève du plaisir. Que tout le monde ait le droit de poster fait partie du jeu, du contrat. [...] Rien de mieux que de s'entraîner pour faire des progrès (« Poster des fan fictions quand on ne sait pas écrire? ", forum Parlons fanfiction).

On a vu, d'autre part, que la volonté de progresser constituait l'une des principales raisons d'écrire mentionnées par les auteurs interrogés par Oger (2012). Enfin, cette dimension de perfectionnement ne se limite pas aux auteurs mais concerne également les lecteurs: l'une des qualités attendues d'un bon bêta-lecteur consiste, en effet, à connaître ses points faibles et à tâcher de les améliorer (Jenkins, 2006a, p. 189).

La centralité de l'apprentissage apparente l'activité à un espace ludique dans le sens où celle-ci ne demande plus d'expertise préalable. Apprendre devient un véritable mode de participation, d'appartenance à la communauté qui peut dès lors être qualifiée de "communauté de pratique" (voir Berry, 2008). Y prévaut un mode informel et collaboratif d'interaction qui n'est pas sans effet sur la manière dont est pratiquée l'écriture.

\section{Les effets de la ludicisation sur la pratique et la jouabilité de l'écriture : l'écriture comme « exercice des possibles»}

En réponse à l'attitude ludique adoptée par les amateurs de fanfictions (dont les différents aspects ont été décrits ci-dessus), la pratique elle-même en vient à développer une certaine jouabilité dont on peut dégager quelques marqueurs observables. Dans un premier temps, l'élaboration de fanfictions peut être comparée au jeu dans la mesure où, comme lui, elle se définit comme un " exercice des possibles " (Genvo, 2011, p. 74) puisqu'elle consiste à réorganiser un univers fictionnel en questionnant une à une toutes ses composantes. Comme l'indique Abigail Derecho (2006, p. 76), même lorsqu'une histoire ainsi représentée semble toucher à sa fin, il reste possible de tout reprendre à zéro à l'aide d'un « what if? ». Par exemple, on peut lire au début d'un chapitre réécrivant un passage du jeu Kingdom Hearts II :

J'ai eu envie d'imaginer comment les choses se seraient passées si Axel avait réagi différemment. Voici ce qui aurait pu arriver si, au lieu de «Tu me manqueras, à moi », il lui avait dit :

- Je pars avec toi (Axel et Roxas dans cent thèmes, chapitre 46).

La réappropriation d'une œuvre à travers l'écriture de fanfictions entraîne, en d'autres termes, l'ouverture du texte original à une plus grande contingence - notion qui constitue selon Thomas Malaby (2007) le fondement même de la jouabilité. Si la fixité du livre, du film ou même du jeu vidéo en tant que game impose une limite à l'activité libre de l'utilisateur (qui, dans les deux premiers cas, se limite à la liberté 
d'interprétation), le détournement de ces textes originaux opère une véritable libération du play:

In fan fiction, there is an acknowledgment that every text contains infinite potentialities, any of which could be actualized by any writer interested in doing the job : fic authors [...] explore situations that the makers of the source text simply cannot, because of the need for continuity and chronological coherence in the source text's universe (and the lack of such a requirement in fan productions) (Derecho, 2006, p. 76). ${ }^{21}$

Cet exercice des possibles libéré des contraintes commerciales et logiques trouve un exemple particulièrement saillant dans l'existence du genre nommé «male pregnancy» (souvent abrégé en "mpreg» ou encore «mp») qui - comme son nom l'indique - rassemble des fictions où sont décrites les grossesses de personnages masculins (pour un exemple concernant Kingdom Hearts, voir Une histoire de rentre dedans).

Plus l'œuvre originale présente de béances exploitables (plus elle contient de «jeu»), plus elle offrira de "prises» aux amateurs (François, 2009, p. 165). Ainsi, l'univers de Kingdom Hearts apparaît comme particulièrement « jouable » : non seulement sa nature de crossover constitue déjà une forme de transgression, mais son intrigue repose, en outre, sur le voyage de personnages entre différents «mondes" (il n'est donc pas étonnant que les fans-auteurs se sentent légitimement invités à concevoir d'autres univers encore inexplorés). Les fanfictions s'adressent à des lecteurs capables de jouer avec ces possibles narratifs et d'envisager les textes dans toute leur potentialité : non comme des ensembles fermés, mais comme les différentes strates d'une expérience ludique continue.

\section{Le texte comme processus ou « work in progress »}

On l'a vu, l'utilisation d'Internet comme support a généralisé, chez les auteurs, une forme «d'écriture par mise à jour " (François, 2009, p. 169) : ceux-ci peuvent diffuser leur texte au fur et à mesure de sa production et en retravailler continuellement les versions antérieures, même après la publication. Cette possibilité confère aux œuvres produites un aspect «temps réel»: le texte est un «work in progress» (Hellekson et Busse, 2006, pp. 6-7) qui ne se solidifie jamais et qui est montré dans son élaboration. En effet, loin d'être dissimulés, les processus d'écriture prennent presque autant de place que les fictions elles-mêmes, envahissant les titres, les espaces de description ou encore les débuts et fins de chaque chapitre.

Ainsi, dans la fiction Axel et Roxas dans cent thèmes, l'auteure émet, avant chaque nouvelle partie, une auto-évaluation sous forme d'astérisques («plus je mettrai d'astérisques, plus je suis satisfaite du chapitre ») et s'engage à se racheter si celle-ci est trop faible ( «si j'en poste de vraiment mauvais, j'en mettrai un deuxième pour rattraper le coup »). L'aspect " en cours » de l'écriture se révèle aussi, lors du vingt-etunième chapitre, nommé "Vacations", à travers la proposition d'un titre alternatif ("Titre alternatif: "L'OXIII à la plage"»), comme si celui-ci était encore en construction et que le choix définitif revenait au lecteur. Dans le même ordre d'idées, un auteur peut exhiber la non-finition de sa production en demandant purement et simplement au public ses desiderata concernant la suite de l'histoire : «Si les lecteurs 
ont une idée pour caser le petit Kadaj, j'écoute » (Kokoro ga nai, chapitre 4), affirme un participant.

Plus généralement, il n'est pas rare de trouver, au sein même des textes, des commentaires sur le style ou l'histoire intégrés par les auteurs ou les bêta-lecteurs :

Un jour, Xemnas les avait envoyés en mission avec Demyx. Mais ce dernier avait cassé une corde de son sitar et s'était barré pour en racheter, ce qui faisait que les deux autres s'étaient retrouvés seuls dans la Forêt des Lotus du Pays des Merveilles. Objectif de la mission: transformer le lapin blanc en Sans-Cœur, dans le but de créer un simili ultrarapide (Oui, je sais, mais fallait bien trouver quelque chose !)

[En fin du chapitre 30 :]

Rangeant dans un coin de sa tête ce qui venait de se passer, Roxas avait ouvert un couloir des Ténèbres pour retourner à l'intérieur de la Forteresse. Il avait ramassé le corps inerte de son ami en tâchant d'ignorer la chaleur brûlante qu'il dégageait, et l'emporta.

Ooooooooh mais NON !!!! Nan mais nan kewa, c'est quoi c'te fin ?! u_u

...

ARIA !!! VIENS ICI FAUT QU'ON PARLE !!!

(Note de la bêta-lectrice en colère. Protégez-moi ! [...])

(Axel et Roxas dans cent thèmes, chapitres 24 et 30)

Roxas se rassit sur sa chaise (on va dire qu'il y avait une chaise, ben oui il va pas s'asseoir par terre le pauvre)

- Pas question que je fasse mes excuses à ce truc, éloigne-le de ma tête! S'écria Roxas, avec un peu de retard (nda: Je peux pas faire parler tout le monde en même temps!)

(My Sweet Dangerous Cat, chapitres 5 et 6)

Cette dualité des textes - qui combinent sans les hiérarchiser des passages de fiction au premier degré et des métacommentaires - invite à envisager l'espace de publication des fanfictions comme un lieu hybride, à la fois salon d'exposition (les œuvres sont diffusées) et atelier d'artiste (le processus de création est encore en cours). Le partage d'un tel espace par les différents participants permet de mieux cerner la dimension collaborative de cette forme d'écriture : le regard du lecteur portant non seulement sur le texte produit, mais aussi sur l'acte de production lui-même ${ }^{22}$.

D'autre part, Internet fait de l'écrit un processus en introduisant une certaine instantanéité dans la publication : « fan fiction [...] is a cultural performance that requires a live audience; fan fiction is not merely a text, it's an event. [...] there's a kind of simultaneity to the reception of fan fiction, a story everyone is reading, more or less at the same time, more or less together » (Coppa, 2006, p. 239). Selon Coppa, la transparence du style des écrits de fans génère de l'instantanéité en permettant un accès plus direct au contenu: les fanfictions développent plutôt un art de la mise en scène qu'une véritable recherche formelle. Cette nature "événementielle " transparait également dans l'organisation récurrente, au sein des communautés, de concours et de célébrations. Si les fêtes traditionnelles sont souvent l'occasion de produire un texte thématisé (une fiction "spécial Noël », par exemple), les fandoms définissent aussi leurs propres périodes rituelles. Dans le cadre de Kingdom Hearts, les personnages qui, au sein du jeu, appartiennent au groupe nommé « Organisation XIII » sont célébrés par les fans lors de «dates anniversaires" correspondant au numéro qui leur est attribué dans ladite Organisation. Le 11 novembre correspond par exemple au personnage Marluxia, onzième membre du groupe : les auteurs fêtent donc à cette date le "Marluxia Day » en produisant des textes qui le mettent en scène. 
39 La publication fragmentée des fanfictions se double, enfin, d'une forte tendance à adopter un style très haché : non contents de diviser leurs histoires en de nombreux chapitres publiés séparément, les auteurs insèrent aussi très fréquemment des sousdivisions au sein de ces portions de texte (voir par exemple Les Bannis ont droit d'amour, chapitre 1). De telles séparations leur permettent de passer rapidement d'une situation à l'autre sans en décrire les transitions ou encore de changer le point de vue depuis lequel est racontée l'action (la plupart des textes étant en focalisation interne). De cette importante discontinuité découle une forme de lecture qui peut être qualifiée d'« hypertextuelle » (il n'est d'ailleurs pas rare que le texte invite le public à se rendre sur une autre fiction, celle du bêta-lecteur par exemple). Or, de cette lecture structurante (par laquelle une cohérence est imposée à des fragments sans cela dispersés) à la participation active, il n'y a qu'un pas: chaque béance du domaine fanfictionnel est une invitation pour le récepteur à entrer dans le jeu de l'écriture.

\section{Une lecture interactive}

Le support numérique a également suscité le développement d'un des marqueurs les plus explicites de la jouabilité des fanfictions, à savoir l'interactivité. La pratique est en effet un exemple parmi d'autres de "culture participative", dans le sens où elle se fonde sur une réception active et créatrice de la part des consommateurs. Toutefois, si l'écriture de fanfictions constituait déjà une forme de réappropriation avant l'avènement d'Internet, l'utilisation de cet outil n'en a pas moins modifié la portée en fournissant aux amateurs de nouvelles possibilités d'interactions : «l'art numérique engage en effet une expérience doublement perceptive et manipulatoire des œuvres » (Fourmentraux, 2012, p. 14), à laquelle ces mêmes œuvres répondent en restructurant leur format. Autrement dit, le numérique a introduit, dans les textes de fanfictions, une certaine « affordance » (Gibson, 1979) qui incite à la participation.

41 Dans un premier temps, la seule adjonction d'une interface introduit une médiation entre le lecteur et le texte qui n'est pas sans rappeler la logique du jeu. Le choix d'une fiction à lire nécessite dorénavant diverses manipulations : dans le menu du site web, il faut préalablement sélectionner le médium de l'œuvre d'origine (livre, film, bande dessinée, jeu, etc.), puis le fandom désiré (quel livre ? quel film ? etc.) pour enfin diriger sa recherche - au sein de l'importante masse de textes - grâce à l'utilisation de filtres. Sur Fanfiction.net, ceux-ci sont particulièrement nombreux : il est possible de trier les œuvres par langue, par genre, par taille, par date, par « rating» (l'œuvre est-elle tout public? réservée aux plus de seize ans ? etc.), en fonction des personnages mobilisés ou encore par tous ces critères à la fois.

Ensuite, cette lecture organisatrice est appelée à déboucher sur une participation effective. Dans sa version la plus minimale, cette participation prend la forme d'une "review», c'est-à-dire d'un commentaire à portée évaluative qui - s'il n'est pas obligatoire - n'en demeure pas moins une norme (les récepteurs qui ne réagissent pas sont désignés par le terme plutôt péjoratif de "lecteurs fantômes »). Les reviews sont constamment sollicitées par les auteurs (il est rare qu'un chapitre ne se termine pas sur une demande de critiques) et peuvent avoir un impact direct sur l'évolution du texte : il en est ainsi dans la fiction Puisque la folie te guette où, suite aux demandes incessantes de ses lecteurs (et de sa bêta-lectrice), l'auteure a réintroduit un personnage dont elle avait tenté de se débarrasser. Que ce soit dans les textes, dans leurs présentations ou 
dans les profils des auteurs, la présence d'autrui est constamment reconnue et convoquée (François, 2009, p. 177) à travers des questions, des sondages ou même à travers la figuration de faux dialogues avec le public :

Dans les rues de la Cité du Crépuscule, marchait un vieil homme aux longs cheveux blonds et au regard inquiétant. Oui, je n'aime pas Vexen. Non, j'arrêterai pas de me payer sa poire, j'aime trop ça. Donc Vexen, car c'était lui, avançait d'un pas tranquille sur le pavé orangé par la lumière du soleil couchant (Axel et Roxas dans cent thèmes, chapitre 53).

Notons que la "collection" de reviews par les auteurs a également pour effet de développer une dimension compétitive de l'activité : un texte disposant de nombreux commentaires aura plus de visibilité sur le site et donc plus de chances d'être lu. Cette évaluation chiffrée de la pratique - de nouveau introduite par Internet - constitue un autre signe de sa jouabilité.

La participation des lecteurs peut trouver une forme accrue dans la pratique de la bêtalecture, déjà évoquée plus haut : en modifiant le texte avant sa parution, ces lecteursexperts prennent part à une partie de son énonciation. Enfin, ces différentes formes de lectures critiques aboutissent généralement dans le passage pur et simple à l'écriture : les lecteurs qui font le pas de commenter les fanfictions d'autrui sont très généralement auteurs eux-mêmes.

\section{Les effets de la ludicisation sur la forme : la jouabilité du texte}

Si certaines «traces » de l'utilisation ludique des fanfictions ont déjà été évoquées (les marques de second degré et de réflexivité, la fragmentation des textes, l'exhibition du processus d'écriture, les signes d'interactivité tels que les reviews ou les intrusions des lecteurs), deux d'entre elles - qui se distinguent par leur aspect essentiellement formel - restent à mentionner. La première concerne le rôle central des personnages dans les fictions, tandis que la seconde relève de l'aspect multimédiatique des écrits fanfictionnels.

L'attention portée, dans les textes, aux personnages fictifs et à leur corps (voir Coppa, 2006) est, en effet, particulièrement saillante. Ceux-ci sont presque systématiquement au centre de l'intrigue (on l'a dit: la majorité des textes étudiés sont en focalisation interne et mettent l'accent sur le ressenti des protagonistes) et viennent même parfois s'y substituer, devenant ainsi des sortes de « mascottes » de l'univers dont ils sont issus. Or l'utilisation d'un personnage comme véhicule pour explorer un monde fictionnel rappelle les codes du jeu et, plus particulièrement, ceux du jeu vidéo. Même si elle n'aboutit pas toujours à la construction d'un avatar de papier (le Mary Sue), la manière dont les fans-auteurs manipulent leurs personnages entretient une certaine parenté avec le jeu de marionnettes ou de figurines (décrit par Jenkins comme l'une des premières possibilités offertes à l'enfant de se réapproprier un contenu culturel, cf. Jenkins, 2002, p. 364). À nouveau, la forme des fanfictions semble donc répondre à une attitude relevant initialement du play.

47 En deuxième lieu, bien que le format des fictions abordées soit bel et bien textuel, celuici n'en demeure pas moins déterminé par le support numérique sur lequel il s'inscrit. Plusieurs recours stylistiques couramment employés révèlent effectivement l'existence d'un «imaginaire multimédiatique». Par exemple, il est courant que les auteurs 
proposent à leur public une liste de chansons à écouter durant la lecture : dans Les Chroniques de l'Héritier Déchu, l'auteure en vient même à attribuer un titre et une musique à chaque phase de l'histoire, comme s'il s'agissait de pistes sur une bande originale. Dans le même ordre d'idées, nombre d'amateurs figurent le médium musical au sein de leurs textes en y insérant des paroles de chansons (voir pour exemple Les Bannis ont droit d'amour). Cette pratique est d'ailleurs considérée comme un genre à part entière, celui des « song fics".

La dimension multimodale des fanfictions se marque, en outre, dans la relation que cette pratique entretient avec le fanart (dans son sens restreint, ce terme renvoie à la production d'œuvres picturales inspirées de fictions préexistantes). Les participants aux deux activités (quand ils ne se confondent pas) collaborent bien souvent à l'enrichissement du fandom ${ }^{23}$.

49 À travers les jeux stylistiques, c'est également le langage numérique lui-même qui est fréquemment représenté. L'utilisation des smileys ou l'omniprésence de traits oraux dans les textes (allongement des voyelles, élision de certaines syllabes, usage abondant des majuscules ou des points d'exclamation dans un but d'expressivité, etc.) rappellent directement le français tchaté (tel qu'il est notamment décrit par Gruppioni, 2012). La plupart des auteurs n'hésitent pas, d'autre part, à utiliser les styles de polices (le gras et l'italique, principalement) et les signes typographiques (astérisques, barres obliques, underscores, etc.) qui sont mis à leur disposition par les logiciels de traitements de texte. La fanfiction ne se contente donc pas de transgresser les limites des œuvres ou la séparation entre auteur et lecteur: elle déborde des frontières qui distinguent les différents médias, assurant une continuité de l'expérience malgré la variation des supports. En cela, symptomatique de la culture de la convergence (Jenkins, 2006a), elle s'apparente aussi au jeu qui, comme le souligne Jesper Juul (2005), est une activité par définition transmédiatique. Le numérique n'a donc pas seulement ouvert le texte fanfictionnel à une certaine jouabilité, il l'a aussi rendu apte à représenter un mode actuel de consommation de la culture où l'œuvre ne s'apparente plus à un objet défini mais à un agencement mouvant qui se construit au fil d'une expérience de circulation entre différents textes, différents médias et différents usages.

\section{Conclusion}

51 En envisageant la fanfiction comme phénomène communautaire, comme pratique et comme texte, nous avons tenté de démontrer que cette forme de réappropriation de la culture répondait à une attitude ludique de la part des utilisateurs - un play porté hors de son espace premier par Internet - et qu'elle en gardait des traces. La présence de ces « signes de la jouabilité » dans une activité qui n'est toutefois pas reconnue comme un jeu permet d'entériner la distinction entre les notions de «ludicisation» et de "gamification». Tandis que la première conceptualise le fait que l'appropriation ludique d'un objet y introduit de la jouabilité, la seconde suppose, à l'inverse, que l'introduction de mécanismes propres au jeu dans un dispositif permet de susciter une réponse «ludique » chez l'utilisateur. Or peut-on vraiment considérer que les processus de gamification transforment des objets non ludiques en jeux s'ils ne cherchent au fond qu'à générer une réponse mécanique chez le consommateur (cliquer pour gagner des points, obtenir une récompense, etc.) ? Imiter la rhétorique du jeu ne suffit pas à rendre une structure jouable : 
A game is a game first because someone has been able to communicate it through a system of objects and rules; but second because this structure becomes a game for someone else through the evolution of its understanding, appropriation, maybe diversions, and surely the sharing of this idea. In brief, when it is played (Philipette, 2014, p. 197).

L'exemple des fanfictions fait apparaitre l'importance - parfois négligée - du détournement, de la transgression et de l'appropriation dans l'émergence d'une activité ludique.

D'autre part, la perspective de la ludicisation a permis d'éclairer le phénomène des fanfictions sous un jour nouveau: loin de se résumer à des textes fermés, celles-ci ouvrent la porte à de multiples usages en laissant la place aux velléités ludiques de leurs participants. En engageant ceux-ci dans une posture réflexive et au second degré, en se présentant comme un processus en cours, permissif et interactif, en permettant la manipulation des fictions et les connexions avec d'autres médias, les fanfictions incarnent une version temporairement solidifiée - et ainsi observable - d'une expérience ludique. Ce qu'apporte leur écriture et leur lecture, c'est la reconduction d'une "expérience magique» passée (Châteauvert et Bates, 2002, pp. 90-91), la concrétisation d'une appropriation individuelle de la culture (rendue plus variable par le numérique), et son partage avec la communauté des pairs.

\section{BIBLIOGRAPHIE}

BATESON G. (1977), Vers une écologie de l'esprit, Paris, Éditions du Seuil.

BERRY V. (2008), « Les communautés de pratiques : note de synthèse ", Pratiques de formation:

Analyses, n $^{\circ}$ 4, p. 12-47, en ligne : http://www.academia.edu/1127961/

Les_communautes_de_pratiques_note_de_synthese

BERRY V. (2014), « Romans, mythes et fictions du monde d'Azeroth : Des exhausteurs de jeu », in Barnabé F. et D. Björn-Olav (dir.), Livre et jeu vidéo, Liège, Bebooks (À paraître).

CHÂTEAUVERT J. et T. BATES (2002), « Films et cultistes », in Le Guern P. (dir.), Les cultes médiatiques: Culture fan et œuvres cultes, Rennes, Presses Universitaires de Rennes, pp. 87-95.

COPPA F. (2006), « Writing Bodies in Space: Media Fan Fiction as Theatrical Performance », in Hellekson K. et K. Busse (dir.), Fan Fiction and Fan Communities in the Age of the Internet, Jefferson, McFarland, pp. 225-244.

CRISTOFARI C. (2010), « Lecteur, acteur : la culture populaire revisitée par les fanfictions et les jeux de rôle ", TRANS-, n9, en ligne : http://trans.revues.org/372.

DERECHO A. (2006), « Archontic Literature: a Definition, a History, and Several Theories of Fan Fiction », in Hellekson K. et K. Busse (dir.), Fan Fiction and Fan Communities in the Age of the Internet, Jefferson, McFarland, pp. 61-78. 
DETERDING S., DIXON D., KHALED R. et L. NACKE (2011), « From Game Design Elements to Gamefulness: Defining "Gamification" ", Actes de la conférence présentée au festival MindTrek'11: Envisioning Future Media Environments, Tampere, pp. 9-15.

FLICHY P. (2010), Le sacre de l'amateur : Sociologie des passions ordinaires à l'ère numérique, Paris, Éditions du Seuil.

FOURMENTRAUX J.-P. (dir.) (2012), L'Ère post-média, Humanités digitales et Cultures numériques, Paris, Éditions Hermann.

FRANÇOIS S. (2009), « Fanf(r)ictions : Tensions identitaires et relationnelles chez les auteurs de récits de fans ", Réseaux, n 153, pp. 157-189 en ligne : http://www.cairn.info/revuereseaux-2009-1-page-157.htm

FRANÇOIS S. (2010), « Appropriations et transpositions amateurs des mass-médias sur Internet. Deux études de cas : les fanfictions et les vidéos amateurs ", sur Télécom ParisTech, en ligne : http://perso.telecom-paristech.fr/ maitre/recherche/rapports-Fondation-fev-2011/francois/ document-miparcours-francois.pdf

FRANÇOIS S. (2012), « La migration des fanfictions sur Internet : aller... et retours ? ", in KomurThilloy G. et A. Réach-Ngô (dir.), L'Écrit à l'épreuve des médias : du Moyen Âge à l'ère électronique, Paris, Classiques Garnier.

GENVO S. (2008), «Caractériser l'expérience du jeu à son ère numérique : pour une étude du "play design" », Communication présentée au colloque Le jeu vidéo : expériences et pratiques sociales multidimensionnelles, Québec, en ligne : http://www.ludologique.com/publis/play_design.pdf

GENVO S. (2011), « Penser les phénomènes de "ludicisation" du numérique : pour une théorie de la jouabilité », Revue des sciences sociales, $\mathrm{n}^{\circ} 45$, pp. 68-77, en ligne : http://www.ludologique.com/ publis/Ludicisation_Genvo_S.pdf

GIBSON J. J. (1979), The ecological approach to visual perception, Boston, Houghton Mifflin.

GRUPPIONI É. (2012), «Conversations électroniques et romans par mails : un exemple d'interférence générique ", in Komur-Thilloy G. et A. Réach-Ngô (dir.), L'Écrit à l'épreuve des médias : du Moyen Âge à l'ère électronique, Paris, Classiques Garnier, pp. 419-436.

HELLEKSON K. et K. BUSSE (dir.) (2006), Fan Fiction and Fan Communities in the Age of the Internet, Jefferson, McFarland.

HENRIOT J. (1989), Sous couleur de jouer, Paris, José Corti.

HUIZINGA, J. (1950), Homo Ludens: A study of the play-element in culture, Boston, Beacon Press.

JENKINS H. (1992), Textual Poachers: Television Fans and Participatory Culture. New York, Routledge

JENKINS H. (2002), « The poachers and the stormtroopers: Cultural Convergence in the Digital Age ", in Le Guern, Philippe, dir., Les cultes médiatiques : Culture fan et œuvres cultes, Rennes, Presses Universitaires de Rennes, pp. 343-378

JENKINS H. (2006a), Convergence Culture: Where old and new media collide, New York, New York University Press

JENKINS H. (2006b), Fans, bloggers, and gamers: Exploring participatory culture, Londres et New York, New York University Press

JONES R. (2006), « From Shooting Monsters to Shooting Movies: Machinima and the Transformative Play of Video Game Fan Culture », in Hellekson K. et K. Busse (dir.), Fan Fiction and Fan Communities in the Age of the Internet, Jefferson, McFarland, pp. 261-280. 
JUUL J. (2005), Half-real : Video Games Between Real Rules and Fictional Worlds, Cambridge, MIT Press.

KARPOVICH A. I. (2006), « The Audience as Editor: The Role of Beta Readers in Online Fan Fiction Communities ", in Hellekson K. et K. Busse (dir.), Fan Fiction and Fan Communities in the Age of the Internet, Jefferson, McFarland, pp. 171-188.

LE GUERN P. (dir.) (2002), Les cultes médiatiques : Culture fan et œuvres cultes, Rennes, Presses Universitaires de Rennes.

LOVELUCK B. (2008), « Internet, vers la démocratie radicale », Le Débat, n 151, pp. 150-166.

MALABY T. M. (2007), « Beyond Play. A New Approach to Games », Games and Culture, vol. 2, n², pp. 95-113, en ligne

http://www.academia.edu/239795/Beyond_Play_A_New_Approach_to_Games

OGER É. (2012), Littérature et Internet. La fanfiction: Enjeux littéraires et éditoriaux, Mémoire de master en Littérature et Linguistique françaises et latines, Université Sorbonne Nouvelle - Paris 3, en ligne : http://www.cavi.univ-paris3.fr/phalese/documents/MEMOIRE\%20E. \%200ger\%20version\%20publique.pdf

PHILIPPETTE T. (2014), « Gamification: Rethinking "playing the game" », in M. Fuchs, S. Fizek, P. Ruffino et N. Schrape (dir.), Rethinking Gamification, Lüneburg, Meson press, pp. 187-200.

POLIAK C. F. (2006), Aux frontières du champ littéraire: Sociologie des écrivains amateurs, Paris, Economica.

RAO V. (2008), « Facebook Applications and Playful Mood: the Construction of Facebook as a "third place" ", Actes de la conférence présentée au festival MindTrek: Entertainment and Media in the Ubiquitous Era, Tampere, pp. 8-12.

SCHAEFFER J.-M. (1999), Pourquoi la fiction ?, Paris, Éditions du Seuil.

SIMONNOT B. (2008), «Quand les moteurs de recherche appellent au jeu : usages ou détournements? », Questions de communication, n 14, pp. 95-114, en ligne http:// questionsdecommunication.revues.org/752

STASI M. (2006), « The Toy Soldiers From Leeds: The Slash Palimpsest », in Hellekson K. et K. Busse (dir.), Fan Fiction and Fan Communities in the Age of the Internet, Jefferson, McFarland, pp. 115-133.

TRICLOT M. (2011), Philosophie des jeux vidéo, Paris, Éditions La Découverte.

VIAL S. (2012), « Pour introduire le "playsir" : jouabilité psychique et ludogénéité numérique », Communication présentée au colloque Jeu et jouabilité à l'ère numérique, Paris

\section{ANNEXES}

\section{Sources du corpus}

«Fanfictions payantes sur Amazon », forum Parlons fanfictions, en ligne : https:// www.fanfiction.net/topic/77279/87893853/1/Fanfictions-payantes-sur-Amazon

Aku'Raichuu, My Sweet Dangerous Cat (chapitres 5 et 6), en ligne : https:// www.fanfiction.net/s/4751757/5/My-Sweet-Dangerous-Cat 
Ariani Lee, Axel et Roxas dans cent thèmes, en ligne : https://www.fanfiction.net/s/ 5360298/46/Axel-et-Roxas-dans-cent-th\%C3\%A8mes

Becca86, Les Chroniques de l'Héritier Déchu, en ligne : https://www.fanfiction.net/s/ 4013327/1/Les-Chroniques-de-1-H\%C3\%A9ritier-D\%C3\%A9chu

Epsylon, Les Bannis ont droit d'amour, en ligne : <https://www.fanfiction.net/s/ 4975923/1/Les-Bannis-ont-droit-d-amour>

Gail LLD, Puisque la folie te guette, en ligne : https://www.fanfiction.net/s/5807613/1/ Puisque-la-folie-te-guette

Lady Lawy, Seconde Chance (chapitre 2), en ligne : <https://www.fanfiction.net/s/ 4575438/2/Seconde-Chance>

Le-baka-d-Hiroshima, Une histoire de rentre dedans, en ligne : https:// www.fanfiction.net/s/6537017/7/Une-histoire-de-rentre-dedans

Moira-chan, Crushcrushcrush (chapitre 2), en ligne : https://www.fanfiction.net/s/ 7107693/2/Crushcrushcrush

Ptirt, La genèse du Yaoi, en ligne : https://www.fanfiction.net/s/5957569/1/Lagen\%C3\%A8se-du-Yaoi

Yumeless, Kokoro ga nai, en ligne : https://www.fanfiction.net/s/5874607/1/Kokoro-ganai

\section{NOTES}

1. Voir, pour exemple, les travaux de Rao, 2008; Simonnot, 2008; ou encore l'ensemble des communications présentées lors du colloque Jeu et jouabilité à l'ère numérique organisé par Université Paris 1 - Panthéon-Sorbonne le 8 décembre 2012.

2. "The transformative play [...] allow[s] players to freely traverse the space between the inside and the outside of the magic circle 》 (Jones, 2006, p. 272).

3. Voir François, 2009, pp. 168-169 pour un récapitulatif plus complet des changements apportés par l'utilisation d'Internet comme support.

4. "As a form, fanfictions make intertextuality visible because they rely on readers' ability to see relationships between the fan-writer's stories and the original media source» (Stasi, 2006, p. 121). Vincent Berry (2014) remarque le même type de conflit interprétatif à propos des novellisations de jeux vidéo : selon lui, celles-ci sont plus perçues par les fans comme des « exhausteurs de jeu » que comme des œuvres littéraires.

5. Il s'agit de jeux d'action-RPG dont le premier opus a été développé par Square en 2002 et qui opèrent un crossover (c'est-à-dire une fusion de plusieurs fictions) entre les univers de Final Fantasy et de Disney. La série a actuellement suscité sur Fanfiction.net l'écriture de 70900 textes.

6. Cet hébergeur est le plus connu en raison de son ancienneté (il a été créé en 1998), de son caractère généraliste (il rassemble des fanfictions inspirées de divers supports et rédigées en plus de quarante langues différentes) et du nombre d'histoires qu'il abrite (3 744842 en janvier 2011 selon le site Fan Fiction Statistics, en ligne : http://ffnresearch.blogspot.be/)

7. Jenkins, 1992, 2002, 2006a et 2006b ; François, 2009, 2010 et 2012; Oger, 2012; tous les articles réunis par Hellekson et Busse (2006); Cristofari, 2010.

8. Le terme fandom désigne à la fois la communauté, les activités et les productions amateur (en somme, toute la sous-culture) qui se développent autour d'une œuvre ou d'un univers fictionnel particulier. 
9. Principalement celles de Sébastien François $(2009,2010,2012)$ et d'Élodie Oger (2012) qui, bien qu'elles aient porté sur les fanfictions basées sur l'univers de la saga Harry Potter, offrent des résultats généralisables au corpus présentement étudié. L'ouvrage de Claude Poliak (2006) - qui ne traite pas des auteurs de fanfictions mais des écrivains amateurs «traditionnels » - pourra également servir de contrepoint lorsque le rapport de ces écrits au champ littéraire sera envisagé.

10. Notons au passage que le nom de ce correcteur renvoie directement au domaine ludique en établissant un parallèle avec le rôle du « bêta-testeur ».

11. Comme le montrent les débats nombreux et argumentés qui s'épanouissent sur les forums (voir, pour exemple, le forum Parlons fanfiction, en ligne : https://www.fanfiction.net/forum/ Parlons-fanfiction/77279/) ainsi que le haut degré de conscience qu'ont les amateurs de l'intérêt universitaire pour leur pratique (une fan-auteure a même créé un site destiné à faciliter les recherches sur le sujet : Études fanfiction, en ligne : http://etude.fanfiction.free.fr/).

12. Près de la moitié des participants à l'enquête d'oger (2012) projettent d'envoyer un texte original (pas une fanfiction, donc) aux maisons d'édition.

13. Les déclarations recueillies par Oger (2012, p. 58) vont également dans ce sens : «quand je réfléchis au statut de la fanfiction, je me demande toujours si nous avons intérêt à ce qu'il devienne tout à fait légal. J'ai un peu peur que des auteurs se prennent la grosse tête et veuillent faire payer les lecteurs".

14. Mary-Sue [dont l'équivalent masculin est nommé «Gary Stu »] est le personnage féminin créé par l'auteur [...] qui débarque dans l'univers du héros et règle tous les problèmes d'un clignement de l'œil. Elle est super belle, super intelligente, super forte, ne se laisse pas faire, fait fondre le cœur de tous les garçons aux alentours, étale le méchant d'un claquement de doigt, a un secret super bien caché qui la rend encore plus forte, et bien sûr, pour renforcer son côté mystérieux, peut se targuer d'avoir des origines étrangères. [...] C'est la fille parfaite, l'incarnation de l'auteur(e) qui vole la vedette au héros et ne décolle pas une seule seconde du dessous des projecteurs. Impossible de s'en débarrasser et Dieu sait pourtant combien les lecteurs ne peuvent la saquer... ». Message introductif posté par une auteure dans le sujet « Le cas MarySue » sur le forum Parlons fanfiction, en ligne : https://www.fanfiction.net/topic/77279/52955121/ Le-cas-Mary-Sue. L'introduction d'un Mary Sue dans une fiction est généralement présentée par les praticiens comme une " erreur de débutant».

15. Le yaoi est le nom japonais d'un genre narratif plus communément nommé «slash » par les fans-auteurs et qui a la particularité de mettre en scène des relations homosexuelles entre personnages masculins.

16. Autre tendance allant dans ce sens : les clins d'œil à la communauté intégrés au sein des histoires. Les personnages féminins de la fiction I've Lost my Heart in Winter, par exemple, figurent un certain profil d'auteur en ce qu'elles sont présentées comme de grandes amatrices de yaoi (en ligne : https://www.fanfiction.net/s/5609560/1/I-ve-lost-my-heart-in-winter).

17. L'abréviation « rp » désigne le roleplay (ou « jeu de rôle »), tandis que «UA » signifie « univers alternatif ».

18. Axel et Roxas sont deux personnages de Kingdom Hearts très populaires et fréquemment mis en couple par les auteurs de fanfictions.

19. RokuNo : abréviation désignant le couple formé par les personnages Roxas et Reno.

20. Les sites Fanfiction.fr (en ligne: http://www.fanfictions.fr/) et Fanfic.fr (en ligne : https:// www.fanfic-fr.net/) fournissent divers liens vers des guides d'écriture, des dictionnaires ou des articles visant à aider les auteurs. Les forums regorgent également de ce type de conseils : voir, pour illustration, les sujets «Stratégies pour détecter les répétitions", "Comment bien faire mourir un perso ? » ou encore «Faire une traduction ; parlons technique » au sein du forum « Le Salon des auteurs ", sur Fanfiction.net, en ligne : https://www.fanfiction.net/forum/Le-Salon-desauteurs/80956/ 
21. La non-soumission de la fanfiction à la cohérence scénaristique se révèle notamment dans la remarque de cette auteure qui présente sa fiction - un recueil de textes courts - en signalant qu'ils « [...] ne suivront pas d'ordre chronologique particulier et [qu'ils] pourront se trouver en totale contradiction les uns avec les autres " (Axel et Roxas dans cent thèmes, chapitre 2, en ligne : https://www.fanfiction.net/s/5360298/2/Axel-et-Roxas-dans-cent-th\%C3\%A8mes)

22. Nous devons cette remarque judicieuse à l'un des évaluateurs anonymes de cet article, qui a également souligné que ce mode de création n'est pas sans rappeler le travail de conception d'un jeu vidéo.

23. De nombreux dessinateurs amateurs ont par exemple représenté un personnage tout droit issu de l'imagination des auteurs de "male pregnancy »: Roxel, le soi-disant fils du couple formé par Axel et Roxas, deux personnages masculins du jeu Kingdom Hearts II.

\section{RÉSUMÉS}

Cet article vise à étudier le phénomène de la « ludicisation » à travers l'une de ses actualisations : l'introduction du ludique dans les pratiques d'écriture amateur que sont les fanfictions diffusées sur Internet. Partant de la perspective des " play studies » (Triclot, 2011) et du " play design » (Genvo, 2008), la ludicisation est ici définie comme la généralisation d'une " attitude ludique » observable à travers les traces (les "signes de jouabilité ») qu'elle laisse sur les objets qu'elle s'approprie. Ce travail tente donc de dégager, au sein d'un corpus de fanfictions inspirées de la série de jeux vidéo Kingdom Hearts et des discours qui les encadrent, d'éventuelles marques de l'usage ludique qui en est fait. Les changements impliqués dans la pratique par l'utilisation du support « ludogène » qu'est le numérique (Vial, 2012) seront également envisagés (celui-ci en accroît-il la jouabilité ?). Les fanfictions seront successivement appréhendées en tant que phénomène communautaire, en tant que pratique d'écriture et enfin, en tant que textes.

This paper aims for studying the ludification phenomenon through one of its realizations: the introduction of play in the writing of fan fictions on the Internet. According to the perspective of the "play studies" (Triclot, 2011) and of the "play design" (Genvo, 2008), the ludification is defined here as the generalization of a "playful attitude" observable through the traces (the "signs of playability") it leaves on the objects it appropriates. This work therefore seeks to identify, within a corpus of fan fiction inspired by the videogame series Kingdom Hearts, any marks of the playful use that is made of these texts. The modifications occasioned, in the practice, by the use of digital technology will also be considered (can this "ludogenic" media increase the playability?; Vial, 2012). The fan fiction will successively be considered as a community phenomenon, as a writing practice and, finally, as a set of texts.

\section{INDEX}

Mots-clés : ludicisation, fanfictions, play studies, Internet, jouabilité

Keywords : ludification, fan fictions, play studies, Internet, playability 
AUTEUR

FANNY BARNABÉ

Université de Liège 